\title{
Matter waves in anharmonic periodic potentials
}

\author{
Tristram J. Alexander, ${ }^{1}$ Mario Salerno, ${ }^{2}$ Elena A. Ostrovskaya, ${ }^{1}$ and Yuri S. Kivshar ${ }^{1}$ \\ ${ }^{1}$ Nonlinear Physics Center and ARC Center of Excellence for Quantum-Atom Optics, Research School of Physical Sciences \\ and Engineering, Australian National University, Canberra ACT 0200, Australia \\ ${ }^{2}$ Dipartimento di Fisica, "E.R. Cainiello” Università di Salerno, I-84081 Baronissi (SA), Italy
}

(Received 16 November 2007; published 4 April 2008)

\begin{abstract}
We study anharmonic (optical or magnetic) periodic potentials and demonstrate that they may exhibit unusual features in the band-gap spectra and the nature of the matter-wave Bloch waves and solitons. We reveal that the band gaps may be strongly modified and even close, and show that changing the potential shape may change the symmetry of the gap-edge Bloch waves and affect the symmetry of higher-order gap solitons. We introduce a mixed-gap matter-wave interface soliton which belongs simultaneously to two different spectral gaps and describe interface localized states in chirped periodic potentials. Finally, we generalize our results to two-dimensional periodic potentials and examine the band-gap structure, matter-wave gap solitons, and lattice vortex states.
\end{abstract}

DOI: 10.1103/PhysRevA.77.043607

PACS number(s): 03.75.Lm

\section{INTRODUCTION}

The study of the effects of periodicity has been of central interest in the fields of condensed matter and optics for many years, with many fundamental generic results predicted theoretically and demonstrated experimentally. More recently, the basic concepts of the physics of periodic systems have appeared in atomic physics in the study of Bose-Einstein condensates (BECs) loaded into optical lattices. BECs offer an unparalleled system of control and simplicity for the study of the effects of periodicity. A rapidly growing body of research already surrounds the behavior of BECs in optical lattices, with the lattices described by simple sinusoidal potentials. However, another well-known way to produce a lattice for cold atoms or BECs is to use periodic arrays of magnetic microtraps, and different types of such magnetic periodic lattices have already been suggested [1] and even demonstrated experimentally [2,3]. In addition, an experimental study of variable symmetric and asymmetric periodic optical potentials has recently been initiated for the realization of ratchet-type effects for cold atoms [4]. This provides a strong motivation for studying systematically the effects of changing the lattice shape on the properties of linear and nonlinear matter waves.

Optical lattices have proven to be an extraordinarily successful tool for manipulating and studying BECs. Their success is founded both on the ease with which they can be used to introduce periodicity-induced effects into the condensate and on the precision with which they may be controlled and dynamically manipulated. To date, general periodicityinduced effects such as Bloch oscillations of wave packets and band structure in the spectrum of low-density extended waves have been studied both theoretically [5] and experimentally [6]. In addition, states which exist due to an interplay of the intrinsic matter-wave nonlinearity and the lattice effects have been demonstrated in the form of gap solitons [8]. In general, only the period, depth, and velocity of the lattice have been varied in the examination of physical phenomena. The precise lattice period and depth are critical in allowing the observation of the above phenomena, and dy- namically changing the depth has revealed the superfluidMott-insulator transition [9]. Changing the velocity has proven very useful for manipulating the diffraction properties of the condensate through a change in its quasimomentum [10]. A much less studied aspect of lattices is how the actual shape of the potential affects the properties of the condensate.

While quite different and unusual periodic potentials have been studied in optics and condensed matter physics, in BEC physics the corresponding results are sparse for nonsinusoidal potentials. The Kronig-Penney-type potential has been studied recently due to its tractable nature [11], as has the elliptic potential [12], while a number of designs have been suggested for nonsinusoidal magnetic and optical lattices [1-4].

In this paper, we consider a variable periodic potential introduced some years ago in the context of the sine-Gordon model $[13,14]$ as an example of an anharmonic periodic magnetic or optical potential. It allows us to consider a variety of potential shapes between the two special limits of the Kronig-Penney model (often used in condensed matter physics) and the inverse Kronig-Penney model (analogous to the deep waveguide lattices studied in optics) with the sinusoidal optical lattice as an intermediate case. First, we examine the linear matter waves in the lattice, with the study of the bandgap diagram and Bloch waves. We reveal that for any lattice depth each higher-order gap will close for a particular lattice shape. We identify that the origin of this effect is in the change of the overlap of the different-symmetry Bloch waves with the lattice. We then examine the localized gap states and

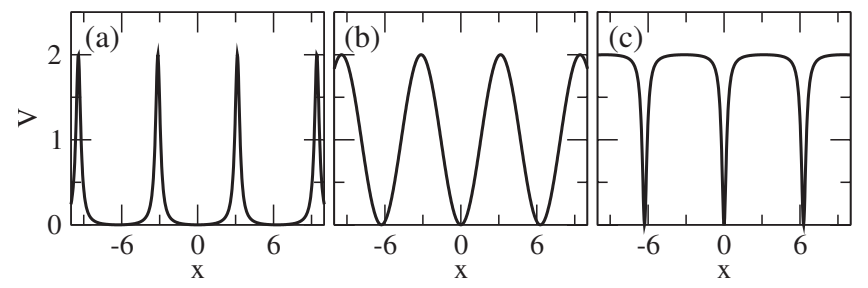

FIG. 1. Examples of the 1D potential (5) for (a) $s=-0.8$, (b) $s$ $=0$, and (c) $s=+0.8$ for $V_{0}=1$. 


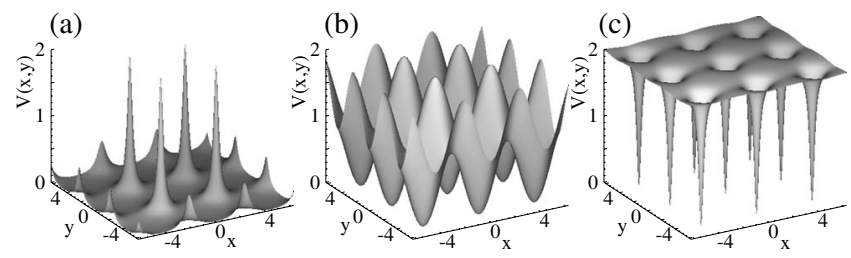

FIG. 2. Examples of the 2D potential (6) for (a) $s=-0.8$, (b) $s$ $=0$, and (c) $s=+0.8$ for $V_{0}=1$.

the matter-wave gap solitons, and demonstrate the importance of both the symmetry of the band-edge Bloch waves and the shape of the potential in determining the shape and stability of the localized solutions. We introduce a higherorder multigap soliton, which occurs when different shape potentials are joined together. Finally, we generalize our results to the case of two-dimensional lattices, examining both the matter-wave gap solitons and vortex states.

The paper is organized as follows. In Sec. II we introduce our model of a variable periodic potential and discuss its limiting cases. Section III is devoted to the analysis of onedimensional lattices, including the linear spectrum for matter waves and gap solitons. In addition, in this section we introduce a localized matter-wave state at the interface created by the same type of the variable lattice with different shapes. In Sec. IV, we present the generalizations of our results to the case of two-dimensional anharmonic lattices. Finally, Sec. V concludes the paper.

\section{MODEL}

The macroscopic dynamics of a Bose-Einstein condensate is described by the Gross-Pitaevskii (GP) equation

$$
i \hbar \frac{\partial \widetilde{\Psi}}{\partial \widetilde{t}}=-\frac{\hbar^{2}}{2 m} \nabla^{2} \tilde{\Psi}+\tilde{V} \tilde{\Psi}+g|\widetilde{\Psi}|^{2} \tilde{\Psi},
$$

where $g=4 \pi \hbar^{2} a_{s} / m$, with $a_{s}$ the $s$-wave scattering length, and $\tilde{V}$ an external potential. We reduce this equation to dimensionless form with the scalings of a standard optical lattice potential, $\tilde{t}=t \omega_{L}^{-1}, \quad(\tilde{x}, \tilde{y}, \tilde{z})=(x, y, z) a_{L}$, and $\tilde{\Psi}=\Psi / \sqrt{8 \pi a_{L}^{2}\left|a_{s}\right|}$ where $a_{L}=d / \pi, E_{r}=\hbar^{2} / 2 m a_{L}^{2}$, and

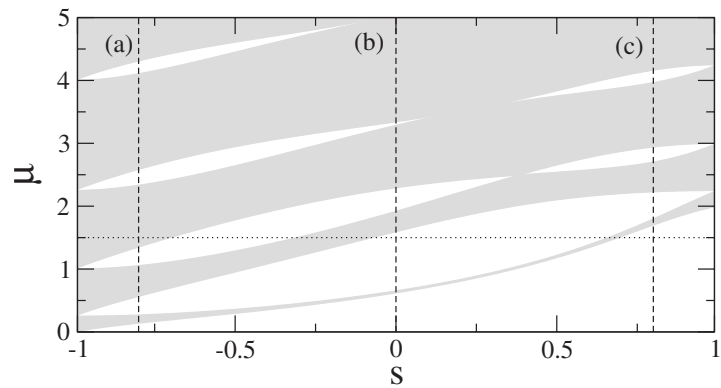

FIG. 3. Appearance of gaps and bands in $\mu$ as $s$ is varied for $V_{0}=1$. Dashed lines correspond to $s$ values of potentials shown in Fig. 1. The horizontal dotted line is at constant $\mu$, highlighting that for different values of $s$ this value of $\mu$ sits in different gaps (see text).

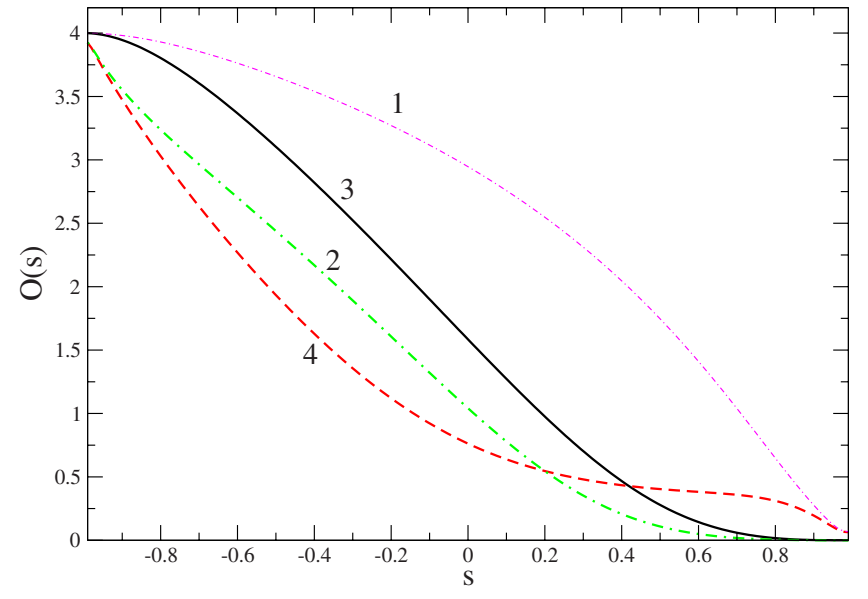

FIG. 4. (Color online) Variation of overlap integral (7) with $s$ for different symmetry Bloch waves at $V_{0}=1$. Numbers correspond to the particular Bloch waves shown in Fig. 5.

$\omega_{L}^{-1}=\hbar / E_{r}$. In this notation $d$ is the period of the optical lattice potential, but half the period of our nonsinusoidal lattice. Our characteristic energy scale $E_{r}$ is the recoil energy of a standard optical lattice, with a characteristic recoil frequency $\omega_{L}$. In the case of a repulsive condensate this gives us the following dimensionless form for the GP equation:

$$
i \frac{\partial \Psi}{\partial t}=-\nabla^{2} \Psi+\frac{\tilde{V}}{E_{r}} \Psi+|\Psi|^{2} \Psi .
$$

The simplest case in which to study the key new effects of changing lattice shape is a reduced-dimensionality model of a condensate. To this end we assume that the condensate is tightly confined in the transverse dimensions, either in a cigar-shaped trap leading to an effectively one-dimensional (1D) condensate or in a pancake-shaped trap for an effectively two-dimensional condensate [7]. As we are interested in only the effects of the lattice, we further assume that in the weakly confined dimension(s) there is only the lattice and no harmonic confinement. Following this dimensionality reduction we have the effective 1D and 2D GP equations

$$
i \frac{\partial \psi}{\partial t}+D_{N} \psi-V \psi+\sigma|\psi|^{2} \psi=0
$$

where $D_{1 D} \equiv \partial^{2} / \partial x^{2}$ in $1 \mathrm{D}$ and $D_{2 D} \equiv \partial^{2} / \partial x^{2}+\partial^{2} / \partial y^{2}$ in 2D, and the nonlinearity coefficient $\sigma$ depends on the transverse trapping potential.

Throughout this work we are interested in the stationary solutions of Eq. (2) and to this end we follow the standard procedure and introduce a chemical potential $\mu$ to account for the nonlinearity-induced phase shift of the stationary solutions. Substituting $\psi(\mathbf{x}, t)=\phi(\mathbf{x}) \exp (-i \mu t)$ into (2) we obtain the time-independent GP equation

$$
D_{N} \phi+\mu \phi-V(\mathbf{x}) \phi+\sigma|\phi|^{2} \phi=0 .
$$

In Sec. III we consider the case of a one-dimensional external periodic potential with variable shape to mimic the effects of the possible nonsinusoidal magnetic lattices. To 

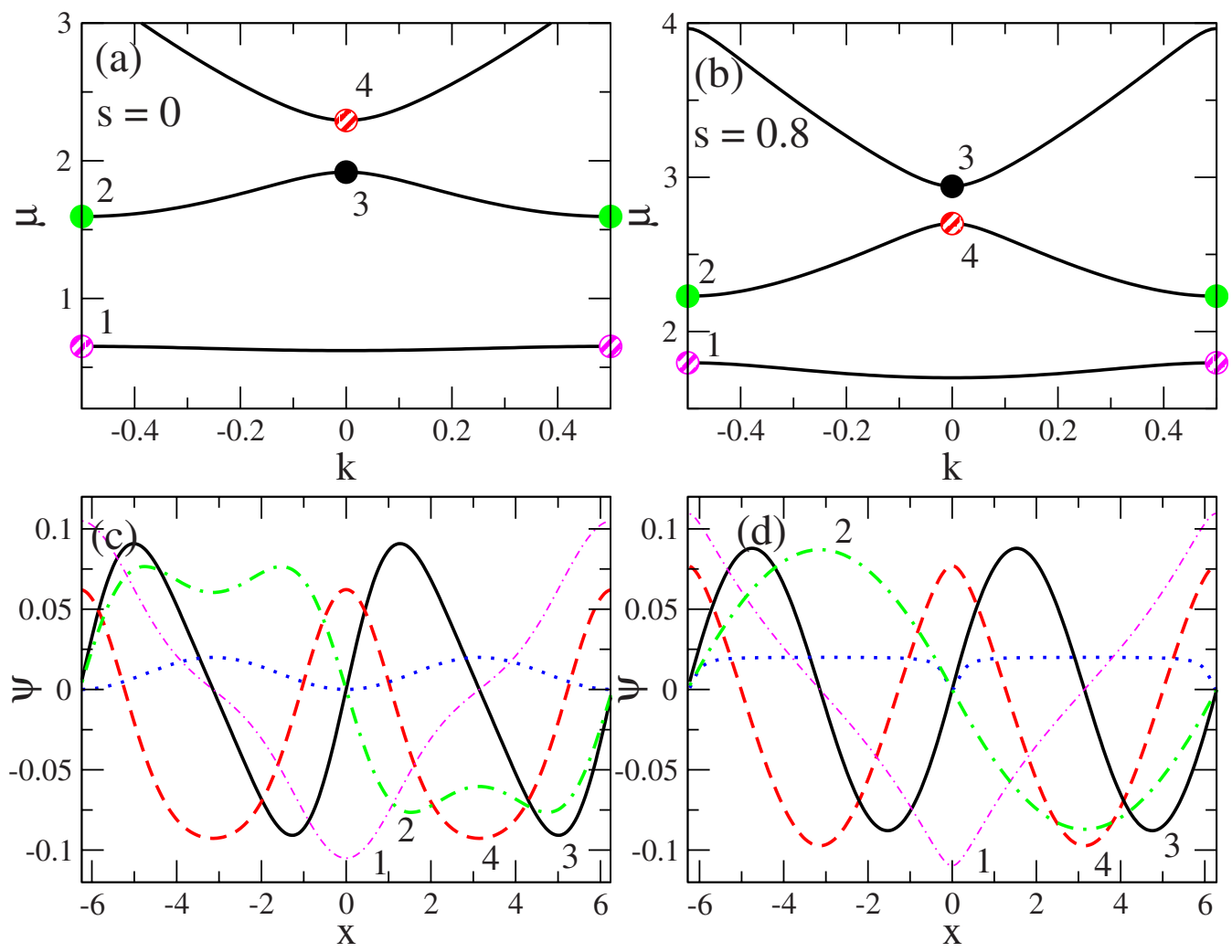

FIG. 5. (Color online) (a) and (b) Band diagram at $s=0$ and $s=0.8$, respectively, for $V_{0}=1$. (c) and (d) First- and second-gap-edge Bloch waves. The dotted line corresponds to the potential. Numbers indicate the band edge in (a) and (b) to which the particular Bloch wave corresponds. The symmetry of the bottom and top second-gap-edge Bloch waves is reversed between the panels.

this end we use a potential first introduced for the sineGordon equation[13]:

$$
V(\mathbf{x})=V_{1 \mathrm{D}}(x)=V_{0}(1+s)^{2} \frac{1-\cos (x)}{1+s^{2}-2 s \cos (x)} .
$$

This particular potential presents a number of advantages for analysis. First, its shape may be scaled by the one parameter $s$, with $-1 \leq s \leq 1$, while the maximum amplitude remains constant at $2 V_{0}$. Second the shape varies from a series of infinitely narrow maxima in the limit $s=-1$ to a series of infinitely narrow wells for $s=1$ with the sinusoidal potential as an intermediate case at $s=0$ (see Fig. 1). Thus we may smoothly examine a wide range of potential shapes.

In Sec. IV we generalize this 1D potential to the case of 2D lattices using the potential

$$
\begin{aligned}
V(\mathbf{x})= & V_{2 \mathrm{D}}(x, y)=V_{0}(1+s)^{2} \\
& \times \frac{1-\cos \left(k_{x} x-k_{y} y\right) \cos \left(k_{x} x+k_{y} y\right)}{1+s^{2}-2 s \cos \left(k_{x} x-k_{y} y\right) \cos \left(k_{x} x+k_{y} y\right)},
\end{aligned}
$$

which for $k_{x}=k_{y}=1 / \sqrt{2}$ gives a square lattice. As in the 1D case, this potential varies between infinitely narrow maxima and minima for $-1 \leq s \leq 1$, as can be seen in Fig. 2 .

Periodic potentials are well known for modifying the linear dispersion relation, even to the extent of introducing gaps in the energy spectrum for which no linear waves exist. From Bloch's theorem, solutions of (4) with a periodic potential and $\sigma=0$ satisfy the relation $\phi(\mathbf{x})=\phi_{b w}(\mathbf{x})=U(\mathbf{x}) \exp (i \mathbf{k} \cdot \mathbf{x})$ where $U(\mathbf{x})$ is a function with the same periodicity as the lattice and $\mathbf{k}$ is the Bloch quasimomentum. The energy spectrum ( $\mu$ vs $\mathbf{k})$ of the Bloch waves $\phi_{b w}(\mathbf{x})$ may then be calculated, characteristically giving energy bands and gaps. The first part of our analysis will examine how changing the potential shape affects this spectrum and the shape of the Bloch waves.

A fundamental effect of the interplay of the periodicity and the nonlinearity is the appearance of states which exist

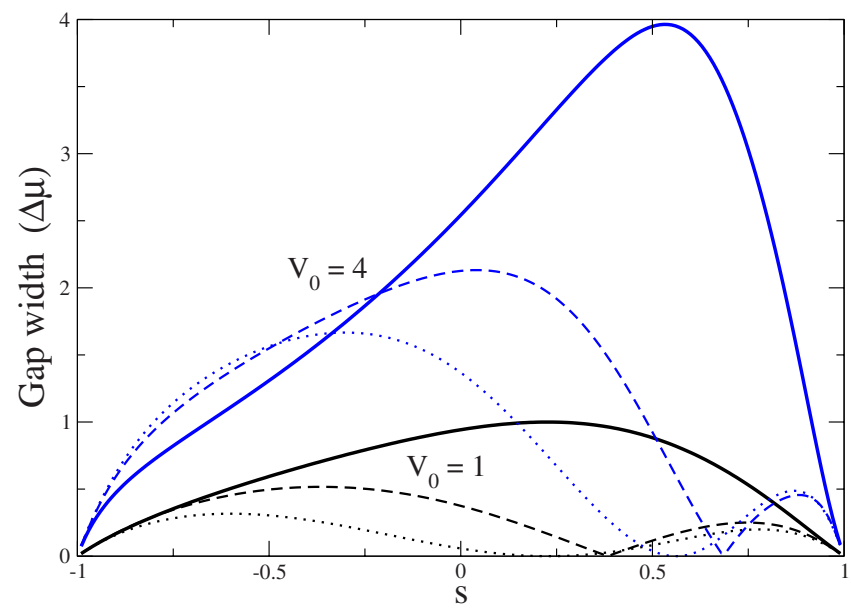

FIG. 6. (Color online) Variation of gap widths with $s$ for first gap (solid line), second gap (dashed line), and third gap (dotted line) for $V_{0}=1$ and $V_{0}=4$. 

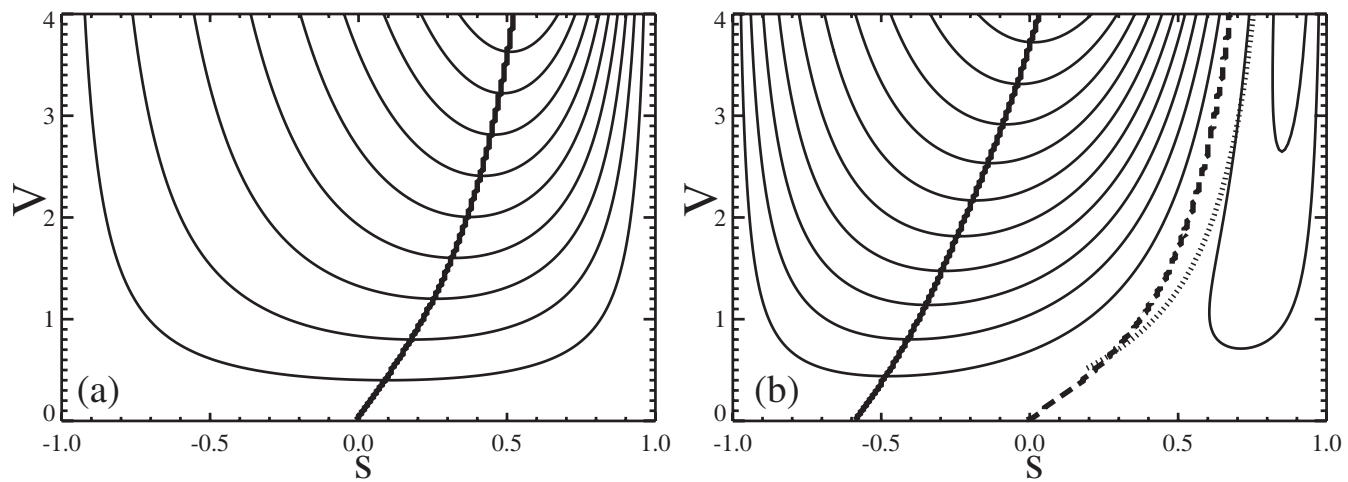

FIG. 7. (a) Change in width of first gap as $s$ and $V_{0}$ are varied. Contour intervals are in steps of 0.4 , and the solid line indicates the maximum width for given $s$ and $V_{0}$. (b) Similar analysis for the second gap; however, now two gaps exist for given $V_{0}$. The dashed line shows the minimum. Contour intervals are in steps of 0.2. The dotted line shows the calculation of gap closure from the overlap, Eq. (7).

inside the linear gaps known as gap solitons. The second part of our analysis will examine how these states change with the potential shape.

\section{ONE-DIMENSIONAL LATTICES}

\section{A. Linear waves}

We begin our analysis by considering the linear states of the 1D periodic potential (5). First, fixing the potential amplitude coefficient $V_{0}=1$ we examine how the gaps in the spectrum change as $s$ is varied. At $s=-1$ the potential is reduced to a periodic array of infinitely narrow maxima, spaced at $x=(2 n-1) \pi$ where $n$ is an integer. In this limit the potential is homogeneous and the gaps close. From the homogeneous spectrum the chemical potential at which each gap closes is thus $\mu_{-1, n}=V_{0} n^{2} / 4$ where $n$ is a positive integer specifying the gap number. Similarly in the limit $s=1$, where the potential is a series of infinitely narrow "holes" on a constant potential $2 V_{0}$, the gaps close at $\mu_{+1, n}=V_{0}\left(2+n^{2} / 4\right)$.
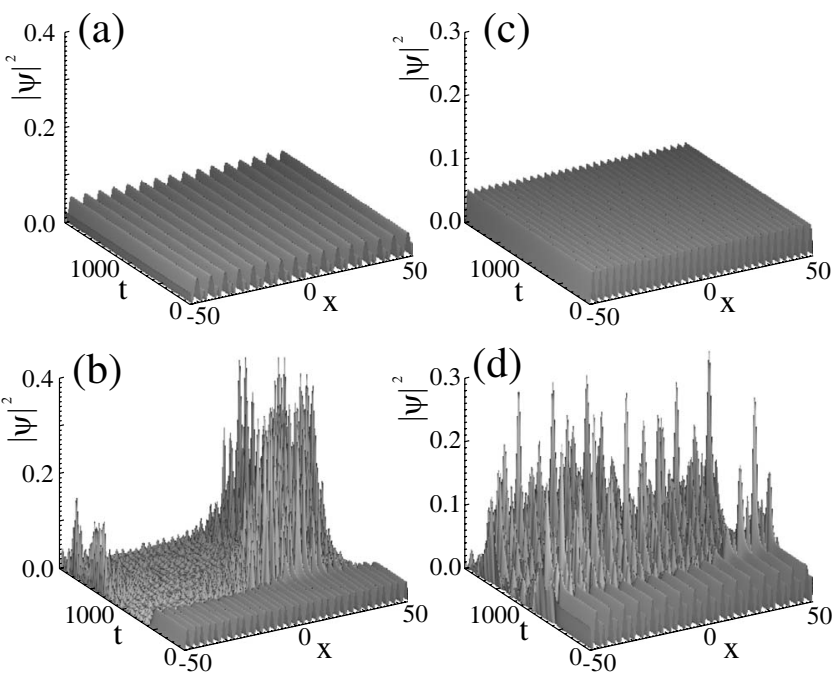

FIG. 8. Low-density second-gap-edge Bloch-wave evolution in the full nonlinear model (3). (a) and (b) $s=0$ ( $\mu=2.33$ and 1.95, respectively) and (c) and (d) $s=0.8(\mu=2.96$ and 2.72, respectively). Top panels: upper gap edge. Bottom panels: lower gap edge.
At $s=0, V(x)=1-\cos (x)$ and thus the potential takes the form of the sinusoidal optical lattice and the gaps can be found by solving the Mathieu equation. Away from these special values the potential is nontrivial in shape and the spectrum must be found numerically using, for example, the eigenvalue solvers in the LAPACK numerical routine package [15]. In Fig. 3 we show the variation of $\mu$ for the gap edges as $s$ is varied. An interesting feature immediately obvious from this figure is that for some special values of $s$ the higher gaps close. Furthermore, it is clear that the optical lattice potential $s=0$ does not yield the largest gaps for either the first gap or the higher gaps. In fact, from inspection it seems that the maximum width of the first gap occurs for some positive $s$,
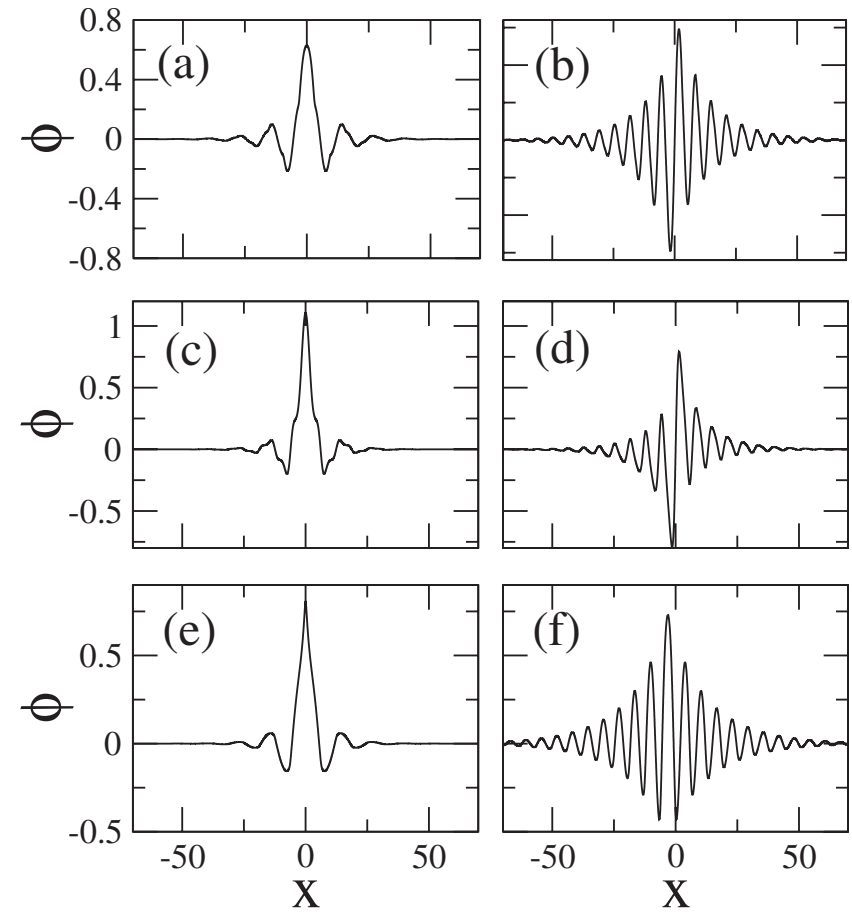

FIG. 9. Gap solitons at (a) and (b) $s=-0.8$ ( $\mu=0.5$ and 1.25, respectively); (c) and (d) $s=0$ ( $\mu=1.55$ and 2.2, respectively); and (e) and (f) $s=0.8\left(\mu=2.15\right.$ and 2.86, respectively) for $V_{0}=1$. Left panels: gap solitons in first gap. Right panels: gap solitons in second gap. 

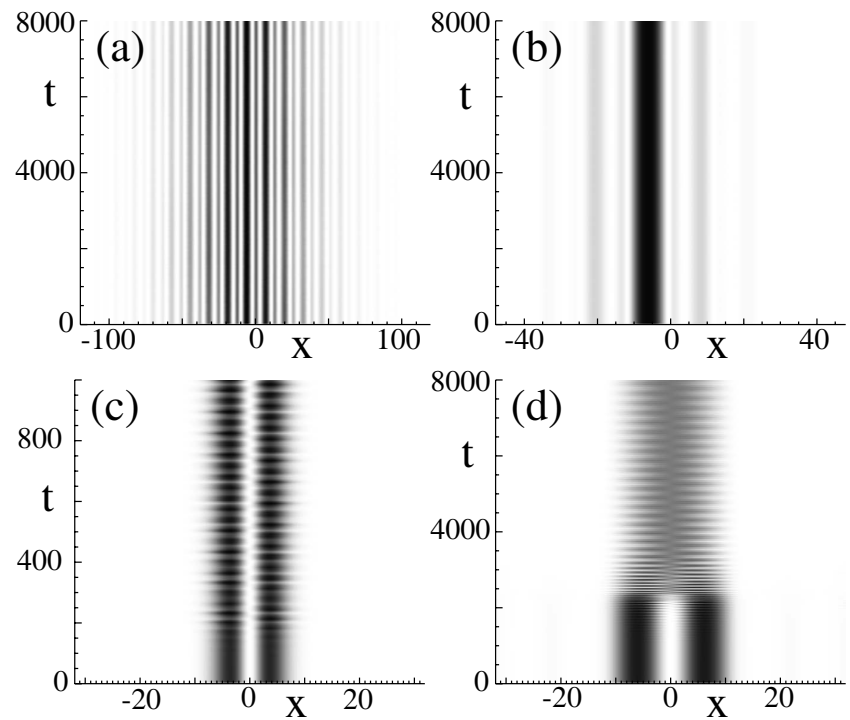

FIG. 10. Evolution of gap solitons in second gap. (a) Secondgap soliton shown in Fig. 9(f) ( $\left.s=0.8, V_{0}=1, \mu=2.86\right)$. (b), (c), and (d) Second-gap solitons in deeper potential $\left(V_{0}=4\right)$ for (b) $s=0.8$, $\mu=8.55$, (c) $s=0, \mu=4.4$, and (d) $s=-0.8, \mu=1.5$.

while for the higher gaps it occurs for different negative $s$ values.

At the simplest level the first gap opens because odd and even waves (relative to the periodic potential and typically waves shifted by a quarter of a wavelength relative to each other) overlap differently with regions of minima and maxima and thus are split in energy. Naturally in a spatially homogeneous potential there is no difference in shifting the linear waves by a quarter wavelength and thus there is no energy splitting. With this in mind we seek to quantify the energy of the gap-edge Bloch waves by examining how they overlap with the potential. We may quantify the change in energy with the overlap integral

$$
O(s)=\int_{-\pi}^{\pi}\left|\phi_{b w}(x)\right|^{2}\left[2 V_{0}-V(x)\right]^{2} d x .
$$

Figure 4 shows the variation of this quantity with $s$ for the first- and second-gap-edge Bloch waves. As can be seen, the second-gap Bloch waves (curves 3 and 4 in Fig. 4) change in relative overlap, while the first-gap Bloch waves do not. The overlap correlates with the energy of the Bloch waves, and thus this crossing indicates a closure of the gap. The dotted line in Fig. 7 corresponds to the closure of the gap calculated using Eq. (7) and shows good qualitative agreement with the numerically calculated gap closure (dashed line).

To understand why the second-gap-edge Bloch waves cross in overlap (and thus lead to a gap closure) while the first gap-edge Bloch waves do not, we need to look at the Bloch waves themselves. As can be seen in Fig. 5 the firstgap-edge Bloch waves always line up with either a minimum or maximum in density on the minima or maxima of the lattice, so changing the shape of the lattice will never lead to a crossing in the overlap. However, the higher-gap-edge Bloch waves have multiple nodes within the one lattice period and so changing the shape of the potential can change
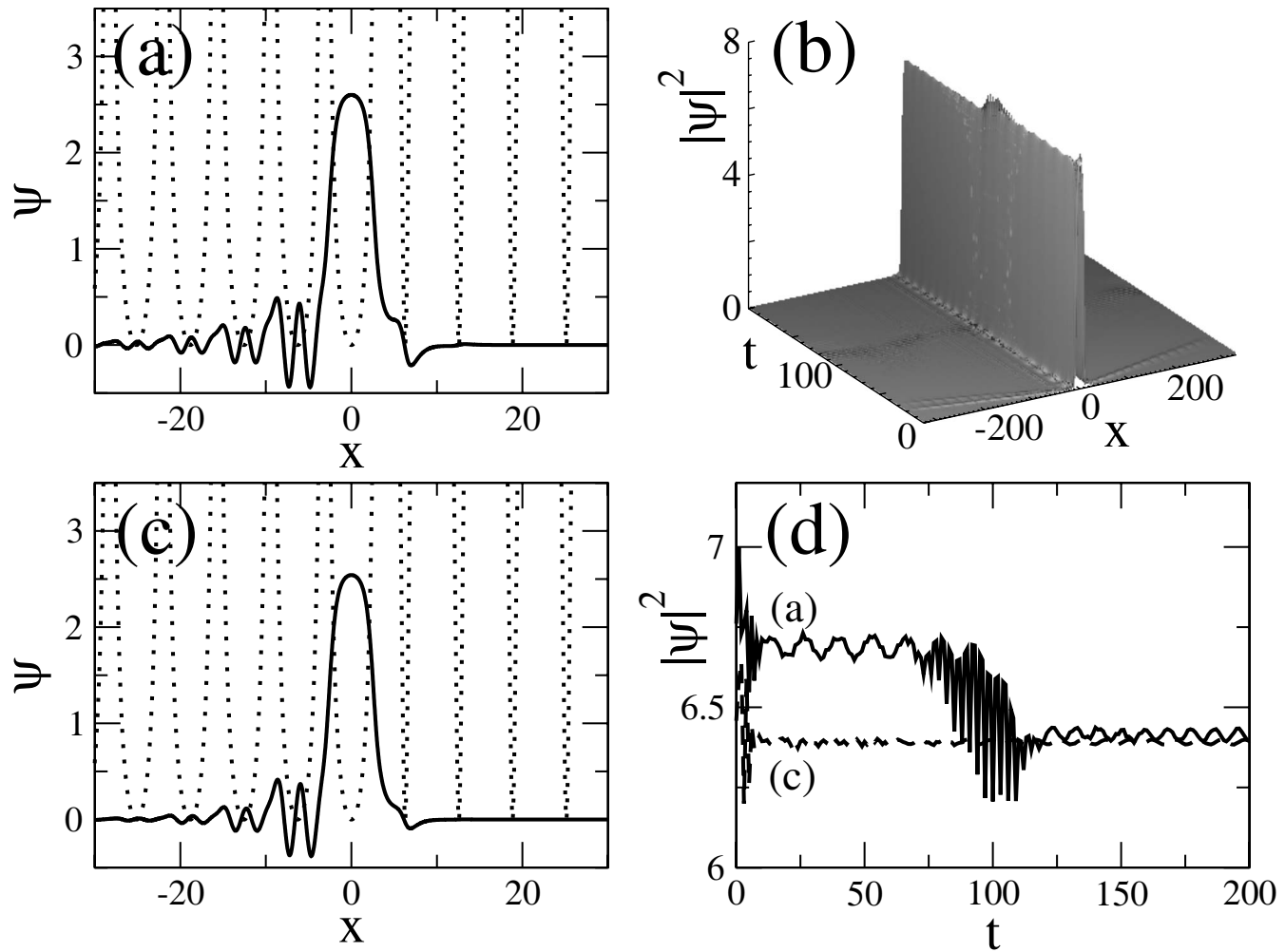

FIG. 11. (a) and (c) Examples of "mixed-gap" solitons existing at interface between $s=-0.7$ and $s=0.7$ potentials (corresponding dotted lines) for $V_{0}=4$ for different $\mu$. (b) Unstable evolution of stationary state shown in (a). (d) Evolution of peak density of the stationary solutions shown in (a) and (c). 


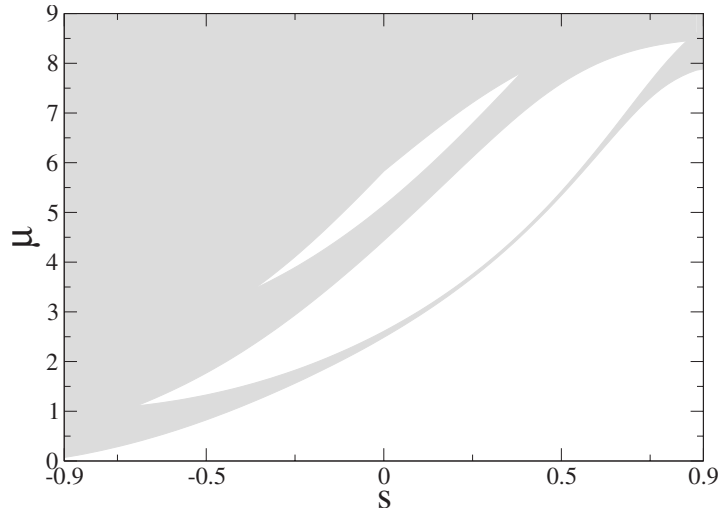

FIG. 12. Dependence of the first and second gaps on $s$ for the 2D potential (6) with $V_{0}=4$.

the overlap. Thus comparing the band-edge Bloch waves on either side of the second-gap closure, we see that they change in relative energy. For $s=0$ the odd Bloch wave is the lower of the two in energy, while for $s=+0.8$ the even Bloch wave is the lower in energy.

In Fig. 6 we examine how the width of the gap varies with $s$ for $V_{0}=1$ and $V_{0}=4$. As can be seen, the $s$ value for which the gaps are maximum is critically dependent on both $V_{0}$ and the number of the gap. The most general features which are immediately clear are that for negative $s$ values higher gaps wider than the first gap are possible, while for larger positive $s$ values the first gap is much larger than the higher gaps. Moving away from a simple sinusoidal lattice the shape of the potential is thus critically important. Depending on the band-gap engineering desired, the relative widths of the different gaps can be dramatically controlled. Figure 7 is a contour plot showing the variation of the widths of the first and second gaps with respect to both $V_{0}$ and $s$. An important point to note is that the dashed line showing the numerical calculation of the second-gap closure can be accurately predicted by examining the overlap (dotted line).

In preparation for our study of the intrinsically nonlinear solutions in the next section, we conclude our Bloch-wave analysis with a numerical investigation of the modulational stability of the second-gap-edge Bloch waves in the lowdensity limit (i.e., for small nonlinearity). Our numerical simulations show that the stability of these gap-edge Bloch waves is determined not by their symmetry, but by whether they are at the lower or upper gap edge and by the sign of the nonlinearity. As we can see in Fig. 8, for repulsive interactions $(\sigma<0)$ the lower-gap-edge Bloch wave is modulationally unstable [Figs. 8(b) and 8(d)] while the upper gap-edge Bloch wave is stable [Figs. 8(a) and 8(c)] even though the density profile of the upper and lower gaps is inverted between [Figs. 8(a)-8(d)]. For attractive interactions $(\sigma>0)$ the opposite is true; i.e., we find that the lower-gap-edge Bloch wave is stable while the upper-gap-edge Bloch wave is unstable. These results are consistent with the modulational instability results found for the first gap, in which for repulsive nonlinearity the lower-gap-edge Bloch wave was found to be unstable and the upper -gap-edge Bloch wave stable [17].
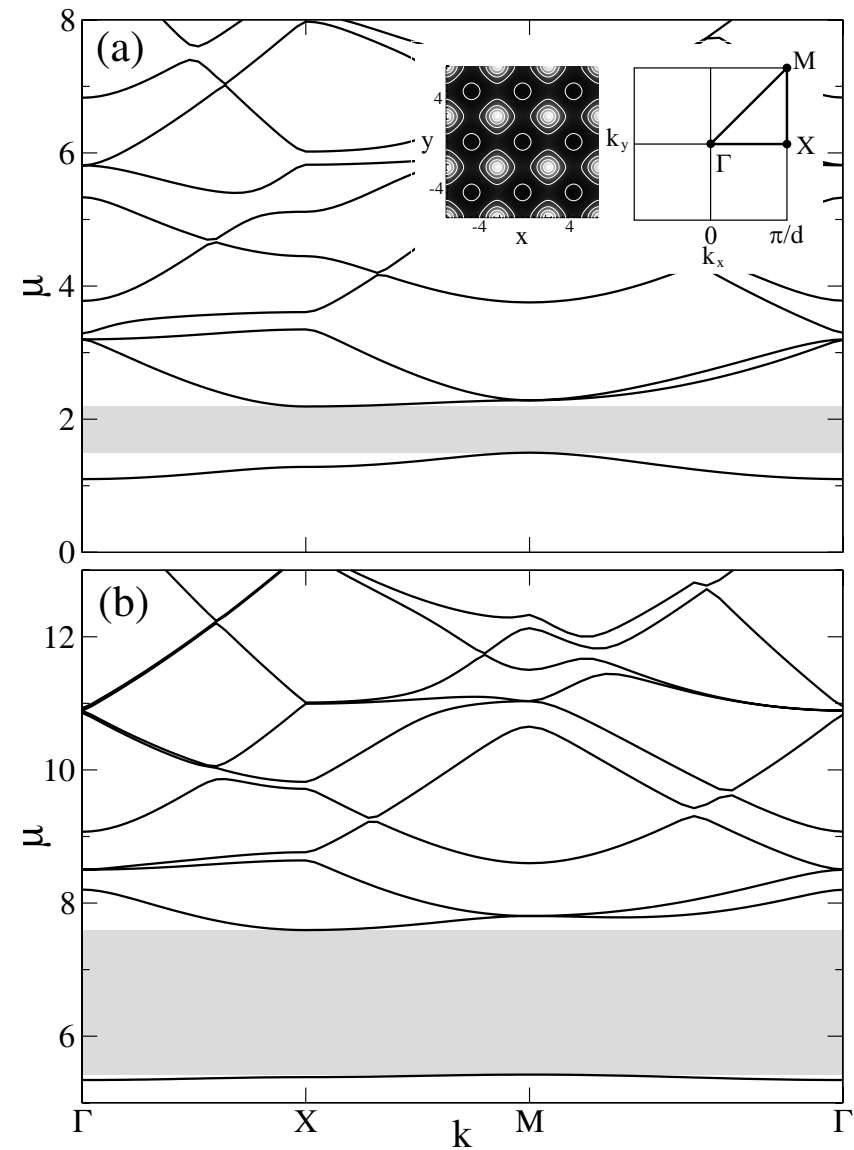

FIG. 13. Band diagram at (a) $s=-0.4$ and (b) $s=0.5$. Shaded regions highlight the first gaps; no higher gaps exist for these $s$ values. The inset shows the $2 \mathrm{D}$ potential at $s=-0.4$ and the corresponding Brillouin zone.

\section{B. Gap solitons}

Having considered the linear effects of changing the lattice shape, we now examine how these results extend into the nonlinear regime by considering one of the most famous periodicity induced nonlinear modes: the gap soliton. Gap solitons in repulsive BECs have been extensively studied in the context of the sinusoidal optical lattice both theoretically [16] and experimentally [8]. They exist as a balance between the anomalous dispersion properties induced by the lattice, characterized by a negative effective mass [8], and the repulsive nonlinearity. They have been studied in discrete systems [18] and for a variety of different potentials in nonlinear optics [19]. We now consider how their properties and appearance change as we change the shape of the potential through the parameter $s$. To this end we solve Eq. (4) with $\sigma=-1$ for a variety of different $s$ values in potential (5). We use a numerical relaxation technique with an appropriate initial guess to find the stationary solutions [20].

In Fig. 9 we show a number of examples with $s$ and chemical potential $\mu$ values indicated as circles in Fig. 3 for $V_{0}=1$. The left panels in the figure show how the gap soliton present in the first gap changes as $s$ is varied. It is clear that while some features in the gap solitons change, such as sharpness of the central peak, the general features remain the 

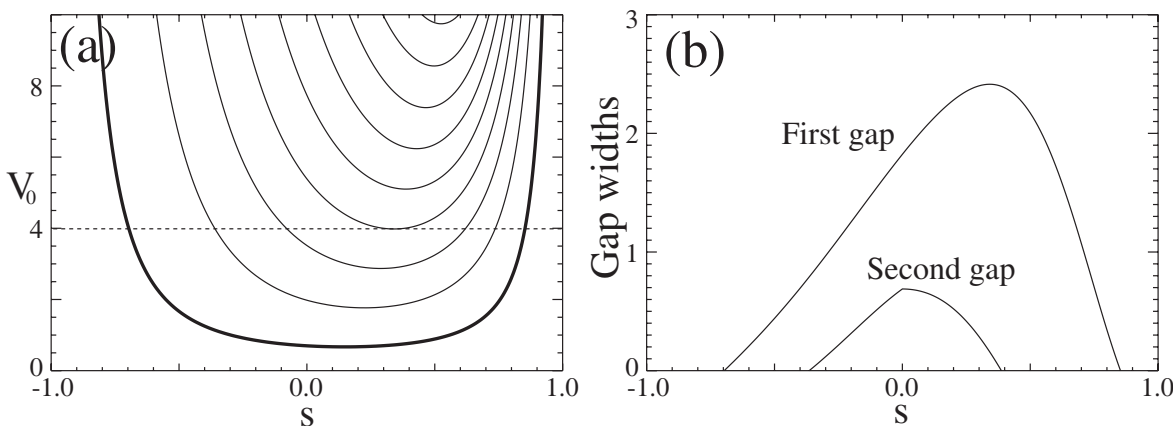

FIG. 14. (a) Gap width as a function of $V_{0}$ and $s$. The thick contour shows the boundary of the existence of the first gap; other contour lines are in gap-width increments of 0.8. (b) Variation of gap width versus $s$ for $V_{0}=4$ [corresponding to the dashed line in (a)] for the first and second gaps.

same. The tails have essentially the same shape, symmetry, and periodicity. The main effects are thus quantitative and directly correlated with the linear results. A larger linear band gap leads to a higher central density and total particle number localized in the lattice. Examining the stability of these solutions using a split-step spectral numerical propagation algorithm, we find that all three solutions shown in Fig. 9 are stable at $t=1000$ with a $0.5 \%$ initial random perturbation.

More significant changes are evident in the gap solitons localized inside the second gap. A fundamental change in the symmetry of these second-gap solitons is evident as we change $s=0$ to $s=0.8$. This is traced back to the change in the symmetry of the lower gap-edge Bloch wave we observed in the linear analysis. While the solitons shown in Figs. 9(b) and 9(d) are both centered on a lattice minimum (on-site) with a node at the very center of the soliton and two equal density adjacent peaks, the solution at $s=0.8$ [Fig. 9(f)] instead is localized on the potential maximum (off-site) and has one central peak. This clearly parallels the symmetry of the lower-gap-edge Bloch waves shown in Fig. 5.

More importantly we find that the stability properties of these gap states change as their symmetry changes. The gap state which bifurcates from the double-peak on-site Bloch wave is unstable to shape changing oscillations [see Figs. $10(\mathrm{c})$ and $10(\mathrm{~d})$ ], typically converging to a different gap state localized in either the first or second gap. The off-site singlepeak second-gap state is, on the other hand, stable (see Figs. 10(a) and 10(b)]. We do, however, find examples of unstable solutions close to the gap edges, as was found for the firstgap solitons in the sinusoidal lattice case [16].

\section{Multigap surface solitons}

Finally we consider an interface gap state which exists in both the first and second gaps. Multigap solitons have already been studied as interface states at the boundary of a homogeneous and periodic medium [21]. In that case the gap solitons existed in the semi-infinite band gap in the homogeneous medium and the first gap in the periodic medium. Detuning of the first gap, so that for a given nonlinearityinduced phase shift (in our language the chemical potential) in the first gap in the periodic medium also sits in the semiinfinite band gap in the homogeneous case, is achieved by increasing the lattice strength. In our case we have an addi- tional "detuning" parameter, the lattice shape parameter $s$. As can be seen in Fig. 3 even for the small lattice depth $\left(V_{0}\right.$ $=1$, potentials with different $s$ have different gaps at the same chemical potential (see the horizontal line of constant $\mu$ in Fig. 3). From this we may predict mixed gap states analogous to the homogeneous and periodic cases except now found at the interface of lattices of different shapes, and more significantly, existing in mixed higher gaps. In Fig. 11 we show examples of a mixed-gap state which exists at the interface between two potentials with $s=-0.7$ on the left and $s=0.7$ on the right. This interface state thus exists in the second gap on the left and the first gap on the right. Our numerical simulations indicate that the stability of these mixed-gap states follows that of the least stable (first or second gap) state. Thus, the solution shown in Fig. 11(a) is unstable due to an instability in the second-gap soliton and converges to the lower-amplitude state as shown in Figs. 11(b) and 11(d). In contrast, the lower-amplitude solution Fig. 11(c) is stable.

\section{TWO-DIMENSIONAL LATTICES}

We now seek to generalize our results to the case of the two-dimensional periodic potential given by Eq. (6). Repeating our analysis of the band structure, we again plot the chemical potential at the gap edges versus the shape parameter $s$. As we can see in Fig. 12, there are a number of marked differences between the one- and two-dimensional cases. First, it is evident that there is no reopening of the higher gaps after they close. As can be seen in Fig. 13, this is a consequence of the additional symmetry directions for the

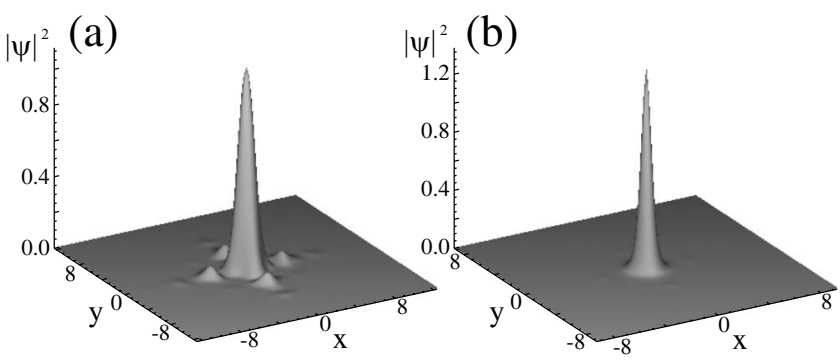

FIG. 15. Gap solitons at (a) $s=-0.4, \mu=1.9$ and (b) $s=0.5, \mu$ $=6$. In both cases, $V_{0}=4$. 
Bloch waves, leading to a much more complicated band diagram. The labels on the $x$ axis correspond to the highsymmetry points of the 2D lattice, shown in the Brillouin zone inset. Thus while quasigaps may reopen in one particular Bloch-wave propagation direction (e.g., the $X$ direction), they may be closed for a different high-symmetry direction. We are interested in complete gaps as these are necessary for nonlinear localization of the condensate wave packet.

We examine in more detail the opening and closing of the gaps in Fig. 14. In Fig. 14(a) we show the dependence of the width of the first gap on both the depth of the potential $V_{0}$ and the shape parameter $s$. It is well known from earlier studies that the first gap in a sinusoidal lattice requires a critical lattice depth before it appears [22]. The widest contour in Fig. 14(a) shows the dependence of the critical lattice depth on the shape parameter $s$. It is evident that for the sharper potentials, near $s= \pm 1$ the first gap may not open at all. This effect is even more pronounced for the second gap. In Fig. 14(b) we see the relative widths of the first and second gaps as $s$ is varied at $V_{0}=4$. Unlike in the 1D case we see that the second gap is always smaller in width than the first and for a given $V_{0}$ appears for a smaller range of potential shapes. The maximum width of the first gap however is found for positive $s$ values, as was the case in the 1D potential.

Having established the linear effects of the lattice shape in 2D, we again turn our attention to the nonlinear modes which may be localized inside the band gaps. Nonlinear localized states are well known to exist in the two-dimensional sinusoidal lattice [23] and are for the most part restricted to the first gap, simply due to the much smaller widths of the higher gaps. We also restrict our analysis to the first gap for this reason. We see in Fig. 15 that changing the shape of the potential essentially changes the localization properties of the soliton. As with the 1D case, we do not see any changes in the symmetry properties of these solitons as the shape of the potential is changed.

A two-dimensional lattice allows the existence of localized nonlinear states with an intrinsic particle circulation which have no one-dimensional equivalent, the so-called gap vortex states [24]. A vortex in the lattice can be regarded as a coupled set of gap solitons, each with a well-defined phase [25]. We are considering a square lattice in which the shape of the minima and maxima may be changed but not the overall symmetry, so we look for the simplest vortex solutions consisting of four lobes with a total $2 \pi$ phase ramp around a singular phase point of zero density. Changing the shape of the lattice allows a change in the effective coupling between the lobes, and this has been shown elsewhere to fundamentally affect both the types of vortices possible in the lattice and their stability [25]. Here we show some examples of the vortex states for different values of $s$. As can be seen in Fig. 16 the localization of the component gap solitons in the lattice fundamentally determines the extent of the vortex. For negative values of $s$ the vortex lobes strongly interact, leading to a more extended vortex state, while for positive $s$ the vortex lobes are much more weakly coupled. Thus using

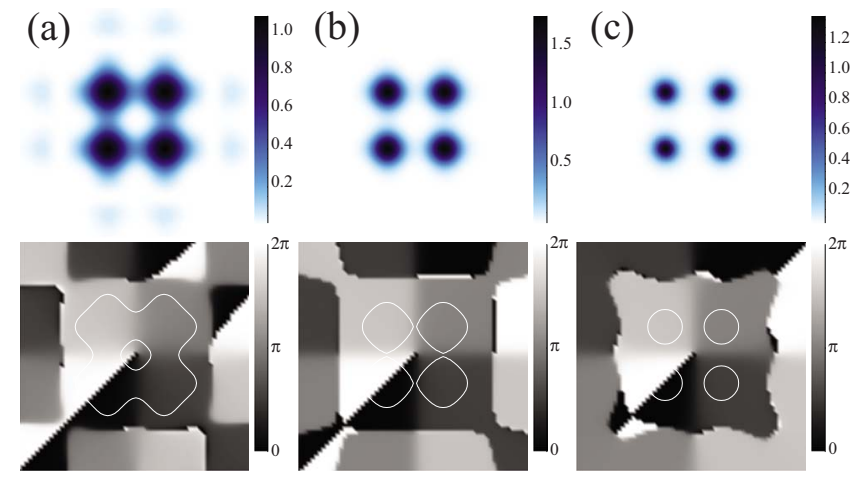

FIG. 16. (Color online) Examples of vortex solitons in the first band gap for (a) $s=-0.4, \mu=1.9$; (b) $s=0, \mu=3.5$; and (c) $s=0.5$, $\mu=6$. In each case the top panels show the density of the wave function and the bottom panels the corresponding phase. Values are given by the color bars on the right. The thin white line in the lower panels outlines a cut at $|\psi|^{2}=0.1$. In each case, $V_{0}=4$.

different shaped lattices is a promising way to induce interesting coupling-dependent dynamical effects such as "charge-flipping", in which perturbations in the vortex density lead to an inversion of the vortex particle circulation [25]. The fundamental nature of the basic vortex is unchanged, however, with all three showing a similar stepped phase profile with a singular point at the center.

\section{CONCLUSIONS}

We have described the properties of linear and nonlinear matter waves in anharmonic (e.g., optical or magnetic) periodic potentials. We have shown that such lattices can demonstrate features in the band-gap spectra; e.g., the gaps in the band-gap spectra may be strongly modified and may even disappear, and the change of the potential shape may modify the symmetry of the gap-edge Bloch waves. Correspondingly, the change of the band-gap spectra modifies the properties and symmetry of matter-wave gap solitons. For the case of the lattice interfaces, we have introduced mixed-gap matter-wave interface solitons which belong simultaneously to two different spectral gaps of different semi-infinite potentials. Finally, we have extended our results to the case of two-dimensional periodic potentials and analyzed the modification of the band-gap structure and matter-wave gap solitons in two-dimensional anharmonic lattices, as well as described matter-wave vortex localized states.

\section{ACKNOWLEDGMENTS}

This work was initiated during the stay of T. Alexander in Salerno, and he thanks the Department of Physics of the University of Salerno for hospitality. M.S. acknowledges partial financial support from the MUR through interuniversity Project No. PRIN-2005: "Transport properties of classical and quantum systems." This work has been supported by the Australian Research Council through the Center of Excellence and Discovery Projects programs. 
[1] J. P. Yin, W. J. Gao, J. J. Hu, and N. C. Liu, Chin. Phys. Lett. 19, 327 (2002).

[2] A. Günther, M. Kemmler, S. Kraft, C. J. Vale, C. Zimmermann, and J. Fortágh, Phys. Rev. A 71, 063619 (2005).

[3] S. Ghanbari, T. D. Kieu, A. Sidorov, and P. Hannaford, J. Phys. B 39, 847 (2006).

[4] G. Ritt, C. Geckeler, T. Salger, G. Cennini, and M. Weitz, Phys. Rev. A 74, 063622 (2006).

[5] K. Berg-Sørensen and K. Mølmer, Phys. Rev. A 58, 1480 (1998).

[6] O. Morsch, J. H. Müller, M. Cristiani, D. Ciampini, and E. Arimondo, Phys. Rev. Lett. 87, 140402 (2001).

[7] Yu. S. Kivshar and T. J. Alexander, in Proceedings of the APCTP-Nankai Symposium on Yang-Baxter Systems, Nonlinear Models and Their Applications, edited by Q-Han Park et al. (World Scientific, Singapore, 1999).

[8] B. Eiermann, Th. Anker, M. Albiez, M. Taglieber, P. Treutlein, K.-P. Marzlin, and M. K. Oberthaler, Phys. Rev. Lett. 92, 230401 (2004).

[9] M. Greiner, O. Mandel, T. Esslinger, T. W. Hansch, and I. Bloch, Nature (London) 415, 39 (2002).

[10] I. Carusotto, D. Embriaco, and G. C. La Rocca, Phys. Rev. A 65, 053611 (2002).

[11] W. D. Li and A. Smerzi, Phys. Rev. E 70, 016605 (2004).

[12] J. C. Bronski, L. D. Carr, B. Deconinck, and J. N. Kutz, Phys. Rev. Lett. 86, 1402 (2001).

[13] M. Remoissenet and M. Peyrard, J. Phys. C 14, L481 (1981).

[14] O. M. Braun and Yu. S. Kivshar, The Frenkel-Kontorova Model: Concepts, Methods, and Applications (Springer-Verlag, New York, 2004), Chap. 3, p. 54, and references therein.

[15] E. Anderson, Z. Bai, C. Bischof, S. Blackford, J. Demmel, J.
Dongarra, J. Du Croz, A. Greenbaum, S. Hammarling, A. McKenney, and D. Sorensen, LAPACK Users' Guide, 3rd ed. (Society for Industrial and Applied Mathematics, Philadelphia, 1999).

[16] P. J. Y. Louis, E. A. Ostrovskaya, C. M. Savage, and Yu. S. Kivshar, Phys. Rev. A 67, 013602 (2003).

[17] V. V. Konotop and M. Salerno, Phys. Rev. A 65, 021602(R) (2002).

[18] A. Trombettoni and A. Smerzi, Phys. Rev. Lett. 86, 2353 (2001); F. Kh. Abdullaev, B. B. Baizakov, S. A. Darmanyan, V. V. Konotop, and M. Salerno, Phys. Rev. A 64, 043606 (2001).

[19] Yu. S. Kivshar and G. P. Agrawal, Optical Solitons: From Fibers to Photonic Crystals (Academic Press, San Diego, 2003).

[20] W. H. Press, S. A. Teukolsky, W. T. Vetterling, and B. P. Flannery, Numerical Recipes in Fortran 77: The Art of Scientific Computing (Press Syndicate of the University of Cambridge, Melbourne, England, 1992).

[21] Y. V. Kartashov, L. Torner, and V. A. Vysloukh, Phys. Rev. Lett. 96, 073901 (2006).

[22] N. K. Efremidis, J. Hudock, D. N. Christodoulides, J. W. Fleischer, O. Cohen, and M. Segev, Phys. Rev. Lett. 91, 213906 (2003); B. B. Baizakov and M. Salerno, Phys. Rev. A 69, 013602 (2004).

[23] B. B. Baizakov, V. V. Konotop, and M. Salerno, J. Phys. B 35, 5105 (2002).

[24] E. A. Ostrovskaya and Yu. S. Kivshar, Phys. Rev. Lett. 93, 160405 (2004).

[25] T. J. Alexander, A. A. Sukhorukov, and Yu. S. Kivshar, Phys. Rev. Lett. 93, 063901 (2004). 